\title{
Video Article \\ The C-seal: A Biofragmentable Drain Protecting the Stapled Colorectal Anastomosis from Leakage
}

\author{
Annelien N. Morks ${ }^{1}$, Klaas Havenga ${ }^{1}$, Henk O. ten Cate Hoedemaker ${ }^{1}$, Rutger J. Ploeg ${ }^{1}$ \\ ${ }^{1}$ Department of Surgery, Division of Abdominal Surgery, University Medical Center Groningen \\ Correspondence to: Annelien N. Morks at a.n.morks@chir.umcg.nl, Klaas Havenga at k.havenga@chir.umcg.nl \\ URL: https://www.jove.com/video/2223 \\ DOI: doi:10.3791/2223
}

Keywords: Medicine, Issue 45, Surgery, low anterior resection, colorectal anastomosis, anastomotic leakage, drain, rectal cancer, circular stapler

Date Published: $11 / 4 / 2010$

Citation: Morks, A.N., Havenga, K., ten Cate Hoedemaker, H.O., Ploeg, R.J. The C-seal: A Biofragmentable Drain Protecting the Stapled Colorectal Anastomosis from Leakage. J. Vis. Exp. (45), e2223, doi:10.3791/2223 (2010).

\section{Abstract}

Colorectal anastomotic leakage $(\mathrm{AL})$ is a serious complication in colorectal surgery leading to high morbidity and mortality rates ${ }^{1}$. The incidence of $A L$ varies between 2.5 and $20 \%{ }^{2-5}$. Over the years, many strategies aimed at lowering the incidence of anastomotic leakage have been examined $^{6,7}$.

The cause of $\mathrm{AL}$ is probably multifactorial. Etiological factors include insufficient arterial blood supply, tension on the anastomosis, hematoma and/or infection at the anastomotic site, and co-morbid factors of the patient as diabetes and atherosclerosis ${ }^{8}$. Furthermore, some anastomoses may be insufficient from the start due to technical failure.

Currently a new device is developed in our institute aimed at protecting the colorectal anastomosis and lowering the incidence of AL. This so called C-seal is a biofragmentable drain, which is stapled to the anastomosis with the circular stapler. It covers the luminal side of the colorectal anastomosis thereby preventing leakage.

The C-seal is a thin-walled tube-like drain, with an approximate diameter of $4 \mathrm{~cm}$ and an approximate length of $25 \mathrm{~cm}$ (figure 1). It is a tubular device composed of biodegradable polyurethane. Two flaps with adhesive tape are found at one end of the tube. These flaps are used to attach the C-seal to the anvil of the circular stapler, so that after the anastomosis is made the C-seal can be pulled through the anus. The C-seal remains in situ for at least 10 days. Thereafter it will lose strength and will degrade to be secreted from the body together with the gastrointestinal natural contents.

The C-seal does not prevent the formation of dehiscences. However, it prevents extravasation of faeces into the peritoneal cavity. This means that a gap at the anastomotic site does not lead to leakage.

Currently, a phase II study testing the C-seal in 35 patients undergoing (colo-)rectal resection with stapled anastomosis is recruiting. The C-seal can be used in both open procedures as well as laparoscopic procedures. The C-seal is only applied in stapled anastomoses within $15 \mathrm{~cm}$ from the anal verge. In the video, application of the C-seal is shown in an open extended sigmoid resection in a patient suffering from diverticular disease with a stenotic colon.

\section{Video Link}

The video component of this article can be found at https://www.jove.com/video/2223/

\section{Protocol}

\section{Fabrication of the C-seal}

The C-Seal is made of biodegradable polyurethane, composed of hard and soft molecular components. The hard component provides the material the desirable elastic and mechanical properties. The hard component consists of polyurethane bindings $\left(\mathrm{HNCO}_{2}\right)$ composed of a diisocyanate (OCN-NCO) and a diol $(\mathrm{HO}-\mathrm{OH})$.

The soft segment determines the degradation behavior of the polyurethane. The soft segment is called the prepolymer and consists of polyetherester chains. The hard segment is incorporated into the soft segment. This incorporation is called 'chain-extension' of the polymerization.

Once these two segments interact, a poly-ether-ester-urethane is synthesized and the polymer is ready to be processed into a C-Seal:

1. The polymer is diluted to a concentration suitable for spray coating.

2. The polyurethane solution is sprayed on a rotating mould to a certain thickness.

3. The mould is placed into a water bath in order to release the C-seal safely from the mould without causing damage. 
4. The C-seal is dried.

5. The C-Seal is packaged in a Tyvek pouch and sterilized using EtO gas.

6. After sterilization, the C-Seal is ready for use.

\section{Surgical Procedure}

The patient has given informed consent for participation in the C-seal study.

1. The patient is positioned in the supine position in stirrups, the abdomen and perineum is disinfected and the patient is covered with sterile drapes.

2. Establishment of pneumoperitoneum or laparotomy via midline incision.

3. Inspection of the liver and abdominal cavity.

4. Mobilization of the sigmoid colon and mobilization of the splenic flexure.

5. In open surgery: division of the sigmoid colon at the level of the proximal resection margin.

6. Right paramedian incision of the peritoneum, opening of the presacral plane; ligation and division of the superior rectal artery and vein.

7. Mobilization of the mesorectum.

8. Division of the rectum distally.

9. In a laparoscopic setting: a suprapubic incision is performed to extract the specimen. Division of the proximal sigmoid colon.

10. The C-seal can be used if the length of the rectal stump is max. 15 centimeters.

11. The bowel diameter determines the stapler diameter; we prefer to use a CEA stapler with a diameter of $29 \mathrm{or} 33 \mathrm{~mm}$.

12. The C-seal and a sterilized $12 \mathrm{~mm}$ stainless steel marble are unpacked on a clean and sterile surface using clean and sterile surgical gloves.

13. Open the $\mathrm{C}$-seal, insert the marble and insert the anvil with the connection pin first. The two flaps in the opening of the $\mathrm{C}$-seal are attached to the anvil using the two strips of adhesive tape.

14. The anvil is advanced in the C-seal so that the connection pin 'punctures' the open pointed end of the C-seal. Gently unfold the C-seal completely and make sure the marble ends up inside the C-seal away from the anvil.

15. Any type of anastomosis (end-to-end, end-to-side, side-to-end and side-to-side) can be performed when using the C-seal; we prefer to create a side-to-end anastomosis. There are multiple strategies to insert the anvil in the proximal bowel loop. The C-seal does not interfere with any of these strategies (open bowel end with pursestring suture/longitudinal bowel incision)

16. Insert the anvil together with the C-seal in de proximal bowel loop by first advancing the C-seal with help of the marble and, if necessary, a blunt forceps. Ensure the tail of the C-seal is placed upwards in the bowel and check that the tail of the C-seal is stretched; check the position of the marble (the marble should be at adequate distance of the anvil). Then penetrate the bowel wall with the anvil's pin and create a pursestring suture around the pin.

17. Gently dilate the anus and insert the stapler in the rectal stump. Connect the anvil with the stapler. Check for torsion of the bowel.

18. Fire the circular stapler. Now, the bowel ends are connected and the C-seal is attached to the staples

19. Gently pull the stapler out of the anus. Do not turn the stapler more than 180 degrees when pulling out (the C-seal may twist). By removing the stapler from the rectum, the $\mathrm{C}$-seal is pulled through the anus. Cut the $\mathrm{C}$-seal at a distance of approximately $4 \mathrm{~cm}$ from the anus.

20. Remove the marble from the rectum. Often the marble comes out together with the C-seal. If not, gently manually remove the marble which is situated between the $\mathrm{C}$-seal and the intestinal wall.

21. Verify the anastomosis. Optionally, perform an air-test by filling the pelvis with a saline solution and inject air into the anal canal (within the Cseal and between the $\mathrm{C}$-seal and bowel). The absence of bubbles confirms a complete anastomosis.

22. If indicated, create a protective stoma.

23. In a laparoscopic setting: exsufflation of the abdominal cavity and trocars extraction.

24. Close the incision(s)

25. Postoperative phase:

Postoperative recovery is according to the ERAS (enhanced recovery after surgery) protocol ${ }^{9}$. The C-seal should be left in situ for at least one week. The patient has no restrictions and can lose stool via the $\mathrm{C}$-seal. When the patient has recovered well and no anastomotic complications are suspected, the C-seal may be cut at the level of the anus. The remaining $\mathrm{C}$-seal will degrade and the patient will lose the $\mathrm{C}$-seal together with the stool via the natural route.

\section{Representative Results}

When the C-seal is applied correctly (figure 2 and 3), it covers the anastomosis. If a dehiscence is formed at the anastomotic site or distal from the anastomosis in the proximal rectal stump, extravasation of feces is prevented since the C-seal covers the defect and directs the fecal matter through the anus. In the postoperative phase, the C-seal protrudes from the anus (figure 3 ).

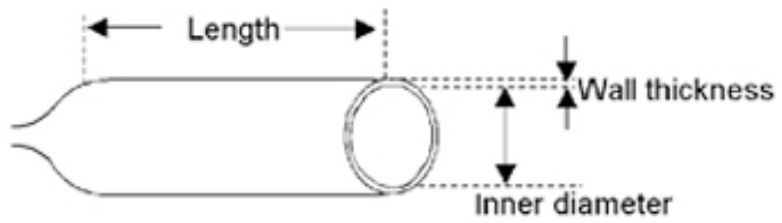

Figure 1: Schematic drawing of the C-seal. 


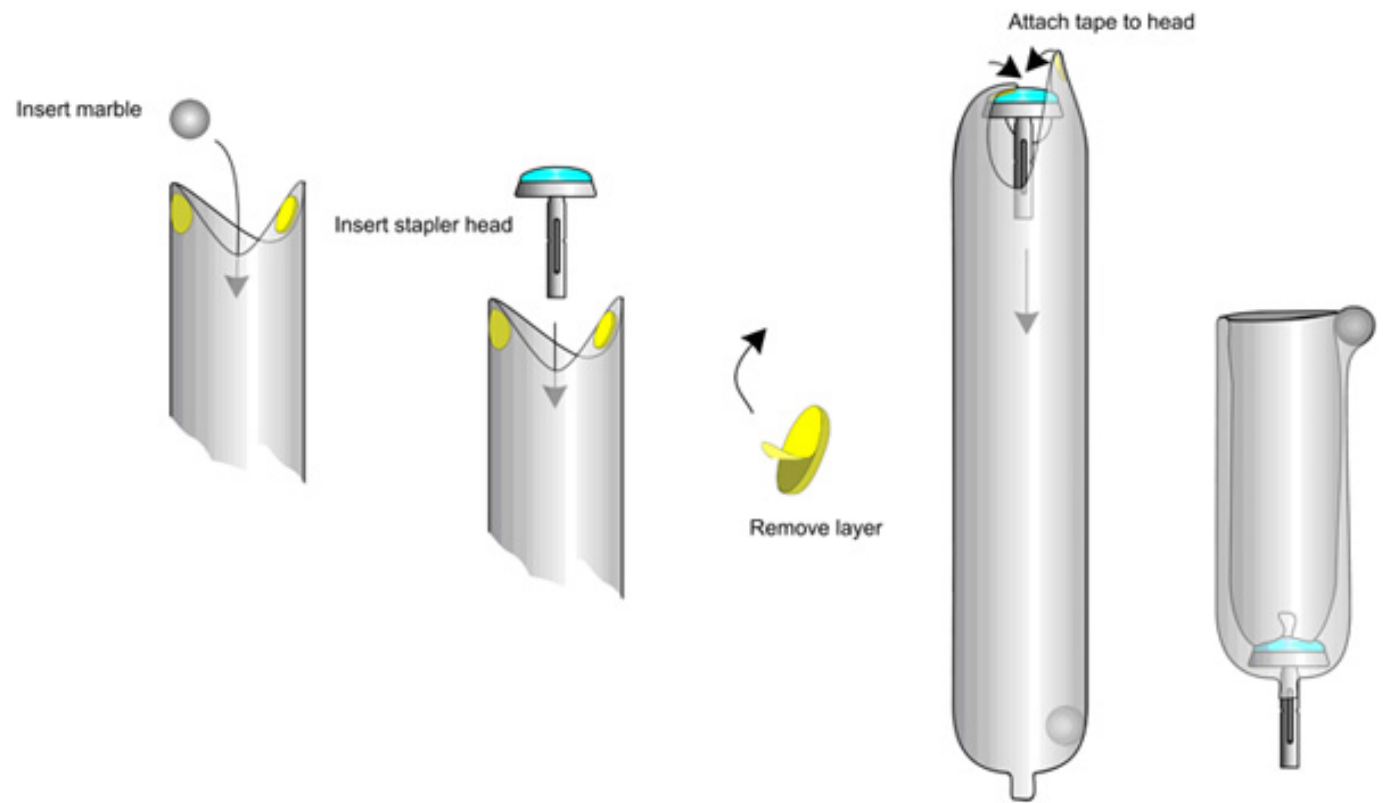

Figure 2: Schematic representation of attaching the C-seal to the stapler head.
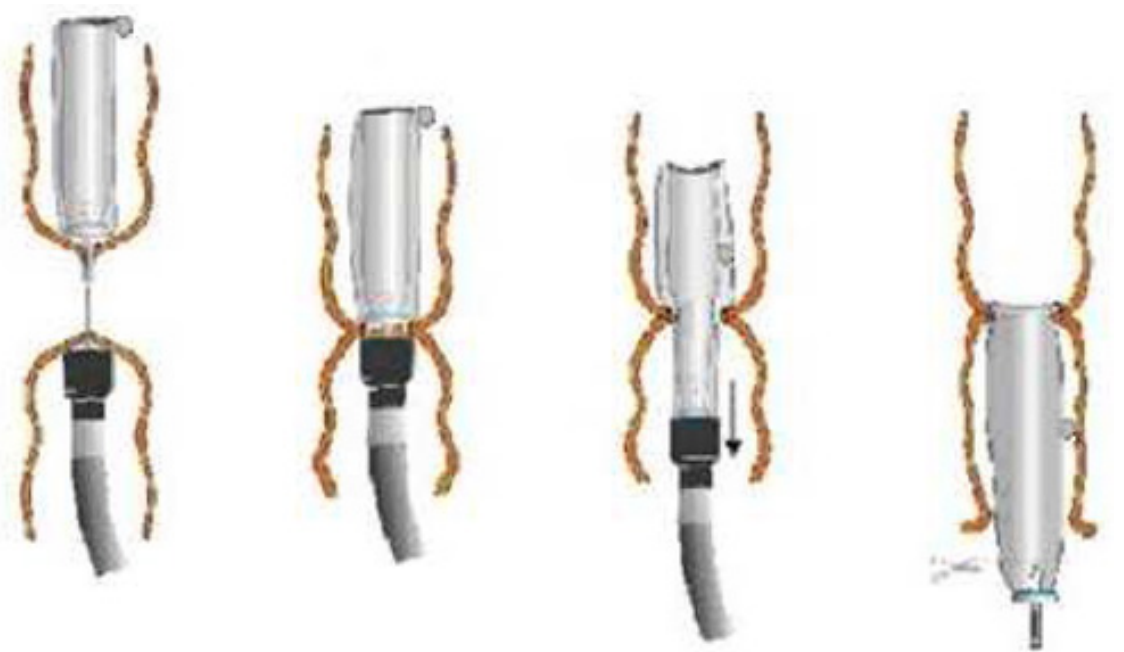

Figure 3: After inserting the stapler-head together with the attached C-seal in the proximal bowel and the stapler in the rectal stump, the stapler is fired and gently pulled through the anus together with the C-seal.

\section{Discussion}

The C-seal is a technique aimed at preventing anastomotic leakage in stapled colorectal anastomoses. It can be used in laparoscopic surgery as well as during a laparotomy. The $\mathrm{C}$-seal does not prevent anastomotic dehiscence; however, it prevents leakage of feces outside the bowel lumen.

When applying the C-seal, extra care should be taken at the moment the C-seal is inserted in the proximal bowel loop. Adequate positioning of the C-seal in the proximal bowel loop is important, since it is theoretically possible to double staple the C-seal. Double stapling of the C-seal occurs when the tail of the C-seal is folded and ends up between the stapler and the stapler head. The marble helps in determining the position of the C-seal in the proximal bowel loop. The marble should be positioned at adequate distance of the stapler head, to guarantee that the C-seal tail is stretched and can not become stapled together with the anastomosis.

The C-seal can be positioned in the proximal bowel loop in different ways. If a side-to-end anastomosis is preferred, we advise to perform this according to the following protocol: The C-seal is inserted via the open bowel end, tail first followed by the stapler head. The connection pin is then penetrated through the bowel wall by stabbing it in the direction of the open bowel end. Subsequently, the open bowel end is closed and a pursestring suture is placed around the connection pin for extra support. The pin is connected to the stapler, the position of the marble is checked and the stapler is fired.

If an end-to-end anastomosis is preferred, the C-seal is inserted in the open proximal bowel end, tail first. A pursestring suture encircles the bowel end around the connection pin. The pin is connected to the stapler, the position of the marble is checked and the stapler is fired. 
In a pilot study initiated by the University Medical Center Groningen, the C-seal was employed in 15 patients undergoing resection of the rectum followed by a stapled anastomoses ${ }^{10}$. No clinical or subclinical AL was diagnosed in these patients. Currently, a phase II study testing the C-seal in another 35 patients is recruiting.

\section{Disclosures}

IRB approval: Procedures involving human subjects have been approved by the Institutional Review Board (IRB) at the University Medical Center Groningen and the Dutch Central Committee of Research inv. Human Subjects (CCMO). No conflicts of interest declared.

\section{Acknowledgements}

The C-seal project is a part of the collaboration between the department of surgery of the University Medical Center Groningen (UMCG) and Polyganics BV. The project was initially supported by a SenterNovem Grant of the Dutch Ministry of Economic Affairs. Intellectual property, commercial interest and patent are held by Polyganics BV. Intellectual interest is held by the UMCG. Polyganics BV has manufactured the devices for this study. The study was performed in the UMCG.

\section{References}

1. Peeters, K.C., et al. Risk factors for anastomotic failure after total mesorectal excision of rectal cancer. Br. J. Surg. 92, 211-216 (2005).

2. Dehni, N., et al. Influence of a defunctioning stoma on leakage rates after low colorectal anastomosis and colonic $\mathrm{J}$ pouch-anal anastomosis. Br. J. Surg. 85, 1114-1117 (1998).

3. Matthiessen,P. Risk factors for anastomotic leakage after anterior resection of the rectum. Colorectal Dis. 8, 366 (2006).

4. Laxamana, A., et al. Long-term results of anterior resection using the double-stapling technique. Dis. Colon Rectum 38, 1246-1250 (1995).

5. Jung, S.H., et al. Risk factors and oncologic impact of anastomotic leakage after rectal cancer surgery. Dis. Colon Rectum 51, 902-908 (2008).

6. Ravo, B., The intracolonic bypass procedure. Int. J. Colorectal Dis. 2, 38-42 (1987).

7. Ye, F., Wang, D., Xu, X., Liu, F., \& Lin, J. Use of intracolonic bypass secured by a biodegradable anastomotic ring to protect the low rectal anastomosis. Dis. Colon Rectum 51, 109-115 (2008).

8. Hawley, P.R. Causes and prevention of colonic anastomotic breakdown. Dis. Colon Rectum 16, 272-277 (1973).

9. Lassen, K., et al. Consensus review of optimal perioperative care in colorectal surgery: Enhanced Recovery After Surgery (ERAS) Group recommendations. Arch. Surg. 144, 961-969 (2009).

10. Kolkert, J.L., et al. Protection of stapled colorectal anastomoses with a biodegradable device: the C-seal feasibility study. Am J of Surg (in Press). 